\title{
Blood Markers of Coagulation, Fibrinolysis, Endothelial Dysfunction and Inflammation in Lacunar Stroke versus Non-Lacunar Stroke and Non-Stroke: Systematic Review and Meta-Analysis
}

\author{
Stewart Wiseman Fergal Marlborough Fergus Doubal David J. Webb \\ Joanna Wardlaw
}

Brain Research Imaging Centre, University of Edinburgh, Edinburgh, UK

\section{Key Words}

Biomarker · Endothelium · Inflammation · Stroke · Lacunar stroke

\begin{abstract}
Background: The cause of cerebral small vessel disease is not fully understood, yet it is important, accounting for about $25 \%$ of all strokes. It also increases the risk of having another stroke and contributes to about $40 \%$ of dementias. Various processes have been implicated, including microatheroma, endothelial dysfunction and inflammation. A previous review investigated endothelial dysfunction in lacunar stroke versus mostly non-stroke controls while another looked at markers of inflammation and endothelial damage in ischaemic stroke in general. We have focused on blood markers between clinically evident lacunar stroke and other subtypes of ischaemic stroke, thereby controlling for stroke in general. Summary: We systematically assessed the literature for studies comparing blood markers of coagulation, fibrinolysis, endothelial dysfunction and inflammation in lacunar stroke versus non-stroke controls or other ischaemic stroke subtypes. We assessed the quality of included papers and meta-analysed results. We split the analysis on time of blood draw in relation to the stroke. We identified 1,468 full papers of which 42 were eligible for inclu-
\end{abstract}

sion, including 4,816 ischaemic strokes, of which 2,196 were lacunar and 2,500 non-stroke controls. Most studies subtyped stroke using TOAST. The definition of lacunar stroke varied between studies. Markers of coagulation/fibrinolysis (tissue plasminogen activator (tPA), plasminogen activator inhibitor (PAI), fibrinogen, D-dimer) were higher in lacunar stroke versus non-stroke although fibrinogen was no different to non-stroke in the acute phase. TPA and PAI were no different between lacunar and non-lacunar stroke. Fibrinogen and D-dimer were significantly lower in lacunar stroke compared to other ischaemic strokes, both acutely and chronically. Markers of endothelial dysfunction (homocysteine, von Willebrand Factor (vWF), E-selectin, P-selectin, intercellular adhesion molecule-1 (ICAM), vascular cellular adhesion molecule-1 (VCAM)) were higher or had insufficient or conflicting data (P-selectin, VCAM) in lacunar stroke versus non-stroke. Compared to other ischaemic stroke subtypes, homocysteine did not differ in lacunar stroke while vWF was significantly lower in lacunar stroke acutely [atherothrombotic standardized mean difference, SMD, -0.34 $(-0.61,-0.08)$; cardioembolic SMD $-0.38(-0.62,-0.14)]$, with insufficient data chronically. Markers of inflammation (C-reactive protein (CRP), tumour necrosis factor-alpha (TNF-a), interleukin-6 (IL-6)) were higher in lacunar stroke versus non-stroke, although there were no studies measuring TNF-a chronically and the sole study measuring IL-6 chroni-

\section{KARGER}

E-Mail karger@karger.com

www.karger.com/ced
(C) 2013 S. Karger AG, Basel

1015-9770/13/0371-0064\$38.00/0
Joanna Wardlaw, FRCP, FRCR, FMedSci

SINAPSE Collaboration, Brain Research Imaging Centre

Neuroimaging Sciences, Western General Hospital

Crewe Road, Edinburgh EH4 2XU (UK)

E-Mail Joanna.Wardlaw@ed.ac.uk 
cally showed no difference between lacunar stroke and non-stroke. Compared to other ischaemic stroke subtypes, there was no difference (CRP) or insufficient or conflicting data (TNF- $a$ ) to lacunar stroke. IL-6 was significantly lower [atherothrombotic SMD $-0.37(-0.63,-0.10)$; cardioembolic SMD -0.52 (-0.82, -0.22)] in lacunar stroke acutely, with insufficient data chronically. Key Messages: Lacunar stroke is an important stroke subtype. More studies comparing lacunar stroke to non-lacunar stroke specifically, rather than to non-stroke controls, are needed. Prospective studies with measurements taken well after the acute event are more likely to be helpful in determining pathogenesis. The available data in this review were limited and do not exclude the possibility that peripheral inflammatory processes including endothelial dysfunction are associated with lacunar stroke and cerebral small vessel disease.

(c) 2013 S. Karger AG, Basel

\section{Introduction}

Lacunar stroke is an important stroke subtype, accounting for one quarter of ischaemic strokes. Its aetiology probably differs from other stroke subtypes, usually being the symptomatic manifestation of small vessel disease rather than large vessel atheroma or cardioembolism.

A previous review [1] of symptomatic lacunar stroke versus mainly non-stroke controls suggests a pathogenic role for endothelial dysfunction but this could simply reflect having an ischaemic stroke in general [2]. Another review [3] found C-reactive protein (CRP), P-selectin and homocysteine to differ significantly between ischaemic stroke (in general) and non-stroke controls, but did not assess levels of blood markers between ischaemic stroke subtypes.

We sought to clarify if differences exist in blood markers between lacunar stroke and other ischaemic stroke subtypes by reviewing the literature for studies measuring coagulation, fibrinolysis, endothelial dysfunction and inflammation. We sought to disentangle the acute phase response by splitting the analysis on timing of the blood draw in relation to the stroke.

\section{Methods}

This review has been prepared in accordance with The PRISMA statement [4]. We extracted data and conducted the metaanalysis in accordance with MOOSE [5], which was modified for our needs using 3 reporting standards [6-8].

Review of Blood Markers in Lacunar Stroke
Search Strategy

We used OVID to search MEDLINE (from 1966) and Embase (from 1980) on July 15th, 2012 using a strategy (see Appendix) developed with advice from the Cochrane Stroke Group (http:// stroke.cochrane.org/).

\section{Inclusion and Exclusion}

We included English language studies published in full measuring blood markers in plasma or serum as follows: tissue plasminogen activator (tPA) as an indicator of fibrinolytic and thrombotic state; plasminogen activator inhibitor (PAI) as an inhibitor of tPA; fibrinogen as a measure of coagulation; D-dimer as a measure of fibrinolysis; homocysteine as a marker of endothelial toxicity; von Willebrand factor (vWF) as a marker of endothelial damage; E-selectin, P-selectin, intercellular adhesion molecule-1 (ICAM) and vascular cellular adhesion molecule-1 (VCAM) as markers of endothelial activation and CRP, interleukin-6 (IL-6) and tumour necrosis factor-alpha (TNF- $\alpha$ ) as markers of inflammation. We excluded studies that did not provide information on blood markers in lacunar stroke and a control group.

\section{Data Extracted}

We extracted data on study population (sample size, age, sex, co-morbidities, current medications); study design; control group (including details on matching for age, sex and covariates where given); blinding of stroke assessor to blood markers and assay investigator to stroke diagnosis; blood markers assessed; time to all blood draws; whether the authors gave detailed methods on how samples were obtained, stored and processed, and results of blood marker levels (both measures of average and spread where given). We recorded method of stroke diagnosis, criteria/system used for stroke subtyping, details of imaging, definition of lacunar stroke and the grade of clinician involved in the stroke diagnosis. We contacted one author [9] to clarify time of blood draw. Some studies drew blood at multiple time points. We recorded data at multiple time points where a relevant comparator was also available. We avoided duplicate publications.

\section{Definition of Lacunar Stroke}

Our gold standard definition of lacunar stroke was all of the following: reference to one of the classical clinical lacunar syndromes; no evidence of cortical dysfunction; imaging (including noting a normal scan does not exclude a lacunar diagnosis), and mention of expertise of person who subtyped the stroke. We relied on the clinical stroke definitions used in the primary papers but tried to harmonize these to a clinical stroke syndrome with support from imaging where available [10].

\section{Meta-Analysis}

We used the Review Manager 5 software (the Cochrane Collaboration) to calculate standardized mean differences (SMD, where data were in a suitable format) using the inverse variance method and a fixed effects model with $95 \%$ confidence intervals (CI). Our forest plots compare lacunar to non-stroke or to nonlacunar stroke (atherothrombotic stroke and/or cardioembolic stroke, as appropriate). Studies [11-13] reporting a geometric mean with $95 \%$ CI were converted to standard deviations (SD) using methods described in the Cochrane Handbook [14].

Not all studies could be meta-analysed due to heterogeneity of reporting, e.g. studies reporting medians with either interquartile 
ranges or minimum and maximum values, as we could not assume normality of distributions. Consequently, tPA, PAI, E-selectin, P-selectin, ICAM, VCAM, CRP and TNF- $\alpha$ were reviewed solely by summary of individual study data. Where studies provided data at more than one acute time point, we meta-analysed only the first time point as this most often corresponded with data for non-stroke comparators. In order to see if an acute phase response affected the results we split our analysis into 'acute' and 'chronic' (bloods drawn up to and after 21 days of stroke, respectively).

\section{Results}

We identified 1,468 full papers. In all, 1,389 titles were excluded following a survey of titles and abstracts, leaving 79 for reading. Of these 10 were excluded (unable to translate) and 32 did not meet inclusion criteria (duplicates, asymptomatic subjects, non-relevant blood markers and no control group). Hand-searching identified a further 5 papers. Therefore, 42 papers were eligible, including 4,816 ischaemic strokes, of which 2,196 were lacunar and 2,500 non-stroke controls (see online suppl. fig. 1 and suppl. table 1; for all online suppl. material, see www.karger.com/doi/10.1159/000356789). In a further 4 papers [15-18] blood was collected prior to stroke. These studies are reported separately (see online suppl. material).

\section{Critical Appraisal of Included Studies}

Over $50 \%$ of studies (22/42) used TOAST [19] to subtype ischaemic stroke. Just 4 studies [11, 20-22] (fewer than $10 \%$ ) met our gold standard definition of lacunar stroke. More than half of the studies (57\%) reported a 'minimal' definition of lacunar stroke; 1 study [23] failed to define lacunar stroke. Most studies recruited patients consecutively (31/42); 1 study recruited non-consecutively [20] and 10/42 did not report on recruitment. Two thirds of studies reported excluding cases based on coexisting disease such as concurrent infection, cancer, inflammatory disease and renal failure. One third of studies did not report on exclusion criteria. Most studies reported matching controls by age and sex to cases (24/42). Some studies matched age only (6/42) and 1 study matched sex only; 3 studies did not report on matching. Matching for co-morbidities varied from study to study. Less than $20 \%$ of studies (8/42) reported blinding of stroke assessor to blood marker values. Fewer still reported blinding of laboratory staff to stroke data (see online suppl. tables 3 and 4 for critical appraisal of included studies).

\section{Plasma Markers}

\section{Coagulation/Fibrinolysis}

Tissue Plasminogen Activator. Here, we included 5 studies [24-28] (251 lacunar strokes) of which 1 study [26] contributed $50 \%$ of the data. There were insufficient data in an appropriate format for meta-analysis. Individual studies suggest tPA was significantly higher in lacunar stroke versus non-stroke controls, both acutely and chronically. Meanwhile, tPA does not appear to differ between lacunar stroke and other stroke subtypes, either acutely or chronically.

Plasminogen Activator Inhibitor. A total of 7 studies were included [24-30] (336 lacunar strokes) but available data did not permit meta-analysis. Jood et al. [26] report levels of PAI significantly higher in lacunar stroke versus non-stroke controls acutely and chronically. They also report lower PAI in lacunar stroke versus atherothrombotic and cardioembolic stroke acutely (significance not given) but not chronically [26]; 4 other studies report no difference between lacunar and non-lacunar stroke acutely [24, 27-29].

Fibrinogen. We included 9 studies [20, 23, 25, 31-36] (622 lacunar strokes) of which 6 permitted a meta-analysis (fig. 1). Fibrinogen showed no difference in lacunar stroke versus non-stroke controls acutely but was significantly higher in lacunar stroke chronically. Fibrinogen was significantly lower in lacunar stroke versus other ischaemic stroke subtypes, both acutely [atherothrombotic SMD -0.37 (95\% CI -0.51, -0.22); cardioembolic SMD $-0.83(-1.15,-0.51)]$ and chronically (fig. 1), although the chronic data comprised only 1 study [32]. In $2[31,34]$ of $3[31,33,34]$ studies that could not be metaanalysed no difference was reported in levels of fibrinogen between lacunar and non-lacunar stroke in the acute phase; the third study [33] did not report on whether its findings were significant.

D-Dimer. Here, 9 studies were included $[25,30,32,34$, 37-41] (364 lacunar strokes) of which 4 could be metaanalysed (fig. 2). D-dimer was significantly higher in lacunar stroke versus non-stroke controls, both acutely [SMD $1.42(1.14,1.69)]$ and chronically [SMD $3.22(2.65$, 3.78)]. D-dimer was significantly lower in lacunar versus non-lacunar stroke, both atherothrombotic [acute SMD $-3.59(-4.06,-3.12)$ ] and cardioembolic [acute SMD $-5.73(-6.38,-5.09)]$, acutely and chronically.

The largest ( $\mathrm{n}=128$ lacunar strokes) of the 5 studies [39] that could not be meta-analysed found no difference in D-dimer between lacunar and atherothrombotic stroke acutely (which disagrees with the meta-analysis) and
Wiseman/Marlborough/Doubal/Webb/ Wardlaw 


\begin{tabular}{|c|c|c|c|c|c|c|c|c|c|c|}
\hline \multirow[t]{2}{*}{ Study or subgroup } & \multicolumn{3}{|c|}{ Lacunar } & \multicolumn{3}{|c|}{ Non-lacunar } & \multirow{2}{*}{$\begin{array}{l}\text { SMD } \\
\text { IV, fixed ( } 95 \% \text { CI) }\end{array}$} & \multirow{2}{*}{\multicolumn{3}{|c|}{$\begin{array}{l}\text { SMD } \\
\text { IV, fixed (95\% CI) }\end{array}$}} \\
\hline & mean & SD & total & mean & SD & total & & & & \\
\hline \multicolumn{11}{|l|}{ Lacunar vs. non-stroke (acute) } \\
\hline Kataoka [32], $2000(<48$ h) & 316 & 15 & 58 & 315 & 16.2 & 32 & $0.06(-0.37,0.50)$ & & 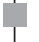 & \\
\hline Beamer [23], 1995 (4 days) & 377 & 133 & 23 & 334 & 39 & 20 & $0.42(-0.19,1.02)$ & & = & \\
\hline Subtotal $(95 \% \mathrm{CI})$ & & & 81 & & & 52 & $0.18(-0.17,0.53)$ & & t & \\
\hline \multirow{2}{*}{\multicolumn{11}{|c|}{$\begin{array}{l}\text { Heterogeneity: } \chi^{2}=0.87, \text { d.f. }=1(p=0.35), I^{2}=0 \% \\
\text { Test for overall effect: } Z=1.02(p=0.31)\end{array}$}} \\
\hline & & & & & & & & & & \\
\hline \multicolumn{11}{|l|}{ Lacunar vs. non-stroke (chronic) } \\
\hline Kataoka [32], 2000 (3 weeks) & 350 & 16 & 58 & 315 & 16.2 & 32 & $2.16(1.62,2.70)$ & & & \\
\hline Salobir [25], 2002 (chronic) & 2.6 & 0.4 & 16 & 2.3 & 0.4 & 47 & $0.74(0.16,1.32)$ & & $\square$ & \\
\hline Subtotal $(95 \% \mathrm{CI})$ & & & 74 & & & 79 & $1.51(1.11,1.90)$ & & 1 & \\
\hline \multirow{2}{*}{\multicolumn{11}{|c|}{$\begin{array}{l}\text { Heterogeneity: } \chi^{2}=12.27, \text { d.f. }=1(p=0.0005), I^{2}=92 \% \\
\text { Test for overall effect: } Z=7.46(p<0.00001)\end{array}$}} \\
\hline & & & & & & & & & & \\
\hline \multicolumn{11}{|l|}{ Lacunar vs. AT (acute) } \\
\hline Zhang [36], 2011 (ON) & 2.96 & 1.04 & 262 & 3.23 & 1.37 & 364 & $-0.22(-0.38,-0.06)$ & & & \\
\hline Kataoka [32], 2000 (<48 h) & 316 & 15 & 58 & 425 & 20 & 41 & $-6.27(-7.25,-5.30)$ & $\rightarrow$ & & \\
\hline Kilpatrick [20], 1993 (48 h) & 3.9 & 1.1 & 9 & 4.1 & 1.2 & 10 & $-0.17(-1.07,0.74)$ & & & \\
\hline Beer [35], 2011 (3 days) & 4.1 & 0.8 & 25 & 4.3 & 1.1 & 19 & $-0.21(-0.81,0.39)$ & & & \\
\hline Beamer [23], 1995 (4 days) & 377 & 133 & 23 & 452 & 91 & 12 & $-0.61(-1.32,0.11)$ & & & \\
\hline Subtotal $(95 \%$ CI) & & & 377 & & & 446 & $-0.37(-0.51,-0.22)$ & & 1 & \\
\hline \\
\hline \multicolumn{11}{|c|}{ Test for overall effect: $Z=4.92(p<0.00001)$} \\
\hline \multicolumn{11}{|l|}{ Lacunar vs. AT (chronic) } \\
\hline Kataoka [32], 2000 (3 weeks) & 350 & 16 & 58 & 480 & 19 & 41 & $-7.46(-8.59,-6.32)$ & 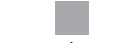 & & \\
\hline Subtotal $(95 \% \mathrm{CI})$ & & & 58 & & & 41 & $-7.46(-8.59,-6.32)$ & $\diamond$ & & \\
\hline \multirow{2}{*}{\multicolumn{11}{|c|}{$\begin{array}{l}\text { Heterogeneity: not applicable } \\
\text { Test for overall effect: } Z=12.90(p<0.00001)\end{array}$}} \\
\hline & & & & & & & & & & \\
\hline \multicolumn{11}{|l|}{ Lacunar vs. CE (acute) } \\
\hline Kataoka [32], 2000 (<48 h) & 316 & 15 & 58 & 340 & 18 & 38 & $-1.47(-1.93,-1.00)$ & & 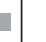 & \\
\hline Beer [35], 2011 (3 days) & 4.1 & 0.8 & 25 & 4.2 & 0.9 & 43 & $-0.11(-0.61,0.38)$ & & 1 & \\
\hline Beamer [23], 1995 (4 days) & 377 & 133 & 23 & 493 & 184 & 6 & $-0.78(-1.71,0.14)$ & & - & \\
\hline Subtotal $(95 \%$ CI) & & & 106 & & & 87 & $-0.83(-1.15,-0.51)$ & & 1 & \\
\hline \multicolumn{11}{|c|}{ Heterogeneity: $\chi^{2}=15.41$, d.f. $=2(p=0.0005), I^{2}=87 \%$} \\
\hline \multicolumn{11}{|c|}{ Test for overall effect: $Z=5.14(p<0.00001)$} \\
\hline \multicolumn{11}{|l|}{ Lacunar vs. CE (chronic) } \\
\hline Kataoka [32], 2000 (3 weeks) & 350 & 16 & 58 & 450 & 20 & 38 & $-5.61(-6.52,-4.70)$ & 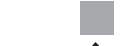 & & \\
\hline Subtotal (95\% CI) & & & 58 & & & 38 & $-5.61(-6.52,-4.70)$ & $\bullet$ & & \\
\hline \multicolumn{8}{|c|}{ Heterogeneity: not applicable } & 101 & & 19 \\
\hline \multirow{2}{*}{\multicolumn{8}{|c|}{ Test for overall effect: $Z=12.11(p<0.00001)$}} & $\begin{array}{cc}-10 & -5 \\
-1 & 0\end{array}$ & 0 & $\begin{array}{ll}5 & 10 \\
\text { Hiaher }\end{array}$ \\
\hline & & & & & & & & in lacunar & & in lacunar \\
\hline
\end{tabular}

Fig. 1. Forest plot - fibrinogen: SMD of blood markers in lacunar stroke versus non-stroke controls and versus non-lacunar stroke controls at different times after stroke. $\mathrm{AT}=$ Atherothrombotic; $\mathrm{CE}=$ cardioembolic; $\mathrm{ON}=$ on admission.

Review of Blood Markers in Lacunar Stroke
Cerebrovasc Dis 2014;37:64-75 DOI: $10.1159 / 000356789$ 


\begin{tabular}{|c|c|c|c|c|c|c|c|c|c|}
\hline \multirow[t]{2}{*}{ Study or subgroup } & \multicolumn{3}{|c|}{ Lacunar } & \multicolumn{3}{|c|}{ Non-lacunar } & \multirow{2}{*}{$\begin{array}{l}\text { SMD } \\
\text { IV, fixed ( } 95 \% \text { CI) }\end{array}$} & \multirow{2}{*}{\multicolumn{2}{|c|}{$\begin{array}{l}\text { SMD } \\
\text { IV, fixed (95\% CI) }\end{array}$}} \\
\hline & mean & SD & total & mean & SD & total & & & \\
\hline \multicolumn{10}{|l|}{ Lacunar vs. non-stroke (acute) } \\
\hline Takano [37], 1992 (1 day) & 115.3 & 15.5 & 23 & 82.1 & 9.1 & 20 & $2.52(1.70,3.34)$ & & - \\
\hline Ageno [38], 2002 (1 day) & 0.67 & 0.08 & 31 & 0.53 & 0.14 & 63 & $1.12(0.66,1.58)$ & & $=$ \\
\hline Kataoka [32], $2000(<48$ h) & 0.7 & 0.1 & 58 & 0.6 & 0.1 & 32 & $0.99(0.54,1.45)$ & & 를 \\
\hline Ilhan [30], 2010 (0-5 days) & 1.36 & 0.62 & 30 & 0.35 & 0.21 & 30 & $2.15(1.51,2.80)$ & & - \\
\hline Subtotal $(95 \% \mathrm{CI})$ & & & 142 & & & 145 & $1.42(1.14,1.69)$ & & 1 \\
\hline \multicolumn{10}{|c|}{$\begin{array}{l}\text { Heterogeneity: } x^{2}=16.89, \text { d.f. }=3(p=0.0007), I^{2}=82 \% \\
\text { Test for overall effect: } Z=10.17(p<0.00001)\end{array}$} \\
\hline \multicolumn{10}{|c|}{ Lacunar vs. non-stroke (chronic) } \\
\hline Kataoka [32], 2000 (3 weeks) & 1.5 & 0.2 & 58 & 0.6 & 0.1 & 32 & $5.20(4.31,6.09)$ & & -1- \\
\hline Takano [37], 1992 (30 days) & 110 & 18 & 23 & 82.1 & 9.1 & 20 & $1.88(1.15,2.61)$ & & \\
\hline Subtotal $(95 \% \mathrm{CI})$ & & & 81 & & & 52 & $3.22(2.65,3.78)$ & & $\diamond$ \\
\hline \multicolumn{10}{|c|}{ Heterogeneity: $\chi^{2}=32.03$, d.f. $=1(p<0.0001), I^{2}=97 \%$} \\
\hline \multicolumn{10}{|c|}{ Test for overall effect: $Z=11.18(p<0.00001)$} \\
\hline \multicolumn{10}{|l|}{ Lacunar vs. AT (acute) } \\
\hline Takano [37], 1992 (1 day) & 115.3 & 15.5 & 23 & 171.3 & 29.4 & 10 & $-2.66(-3.67,-1.65)$ & $-\square$ & \\
\hline Ageno [38], 2002 (1 day) & 0.67 & 0.08 & 31 & 1.34 & 0.21 & 34 & $-4.09(-4.97,-3.22)$ & - & \\
\hline Kataoka [32], 2000 (<48 h) & 0.7 & 0.1 & 58 & 1.7 & 0.4 & 41 & $-3.70(-4.36,-3.04)$ & 드. & \\
\hline Subtotal $(95 \% \mathrm{CI})$ & & & 112 & & & 85 & $-3.59(-4.06,-3.12)$ & $\bullet$ & \\
\hline \\
\hline \multicolumn{10}{|c|}{ Test for overall effect: $Z=15.06(p<0.00001)$} \\
\hline \multicolumn{10}{|l|}{ Lacunar vs. AT (chronic) } \\
\hline Kataoka [32], 2000 (3 weeks) & 1.5 & 0.2 & 58 & 2.3 & 0.4 & 41 & $-2.65(-3.20,-2.10)$ & 口 & \\
\hline Takano [37], 1992 (30 days) & 110 & 18 & 23 & 125 & 20 & 10 & $-0.79(-1.56,-0.02)$ & & \\
\hline Subtotal (95\% CI) & & & 81 & & & 51 & $-2.02(-2.47,-1.58)$ & $\bullet$ & \\
\hline \multirow{2}{*}{\multicolumn{10}{|c|}{$\begin{array}{l}\text { Heterogeneity: } \chi^{2}=14.97, \text { d.f. }=1(p=0.0001), I^{2}=93 \% \\
\text { Test for overall effect: } Z=8.86(p<0.00001)\end{array}$}} \\
\hline & & & & & & & & & \\
\hline \multicolumn{10}{|l|}{ Lacunar vs. CE (acute) } \\
\hline Takano [37], 1992 (1 day) & 115.3 & 15.5 & 23 & 607 & 167.6 & 21 & $-4.16(-5.24,-3.07)$ & - - - - & \\
\hline Ageno [38], 2002 (1 day) & 0.67 & 0.08 & 31 & 2.96 & 0.51 & 34 & $-6.06(-7.24,-4.88)$ & $-\square$ & \\
\hline Kataoka [32], $2000(<48 \mathrm{~h})$ & 0.7 & 0.1 & 58 & 3 & 0.5 & 38 & $-7.06(-8.16,-5.96)$ & $-\square-$ & \\
\hline Subtotal $(95 \% \mathrm{CI})$ & & & 112 & & & 93 & $-5.73(-6.38,-5.09)$ & $\diamond$ & \\
\hline \multirow{2}{*}{\multicolumn{10}{|c|}{$\begin{array}{l}\text { Heterogeneity: } X^{2}=14.01, \text { d.f. }=2(p=0.0009), I^{2}=86 \% \\
\text { Test for overall effect: } Z=17.39(p<0.00001)\end{array}$}} \\
\hline & & & & & & & & & \\
\hline \multicolumn{10}{|l|}{ Lacunar vs. CE (chronic) } \\
\hline Kataoka [32], 2000 (3 weeks) & 1.5 & 0.2 & 58 & 3.2 & 0.5 & 38 & $-4.82(-5.62,-4.01)$ & 들 & \\
\hline Takano [37], 1992 (30 days) & 110 & 18 & 23 & 255 & 40 & 21 & $-4.67(-5.85,-3.48)$ & - & \\
\hline Subtotal $(95 \% \mathrm{CI})$ & & & 81 & & & 59 & $-4.77(-5.43,-4.10)$ & $\diamond$ & \\
\hline \multirow{3}{*}{\multicolumn{8}{|c|}{$\begin{array}{l}\text { Heterogeneity: } \chi^{2}=0.04, \text { d.f. }=1(p=0.84), I^{2}=0 \% \\
\text { Test for overall effect: } Z=14.03(p<0.00001)\end{array}$}} & & \\
\hline & & & & & & & & $-4-2$ & $\begin{array}{l}24 \\
2 \text { Higher }\end{array}$ \\
\hline & & & & & & & & in lacunar & $\begin{array}{l}\text { Higner } \\
\text { in lacunar }\end{array}$ \\
\hline
\end{tabular}

Fig. 2. Forest plot - D-dimer: SMD of blood markers in lacunar stroke versus non-stroke controls and versus non-lacunar stroke controls at different times after stroke. $\mathrm{AT}=$ Atherothrombotic; $\mathrm{CE}=$ cardioembolic. 


\begin{tabular}{|c|c|c|c|c|c|c|c|c|c|c|}
\hline \multirow[t]{2}{*}{ Study or subgroup } & \multicolumn{3}{|c|}{ Lacunar } & \multicolumn{3}{|c|}{ Non-lacunar } & \multirow{2}{*}{$\begin{array}{l}\text { SMD } \\
\text { IV, fixed }(95 \% \text { CI) }\end{array}$} & \multirow{2}{*}{\multicolumn{3}{|c|}{$\begin{array}{l}\text { SMD } \\
\text { IV, fixed }(95 \% \text { CI }\end{array}$}} \\
\hline & mean & SD & total & mean & SD & total & & & & \\
\hline \multicolumn{11}{|l|}{ Lacunar vs. non-stroke (acute) } \\
\hline Parnetti [42], 2004 (NS) & 13.9 & 5.4 & 50 & 8.1 & 2.5 & 152 & $1.68(1.32,2.04)$ & & & - \\
\hline Jeong [44], 2011 (3 days) & 11.3 & 3.8 & 83 & 11.2 & 3.4 & 135 & $0.03(-0.25,0.30)$ & & - & \\
\hline $\begin{array}{l}\text { Khan }[9], 2008 \\
\text { ( } 2-3 \text { days) }\end{array}$ & 16.2 & 11.6 & 152 & 11.8 & 5.7 & 179 & $0.49(0.27,0.71)$ & & - & - \\
\hline $\begin{array}{l}\text { Eikelboom }[11], 2000 \\
\text { (<7 days) }\end{array}$ & 12.7 & 5.577 & 68 & 10.5 & 3.6309 & 205 & $0.52(0.24,0.80)$ & & & - \\
\hline Subtotal (95\% CI) & & & 353 & & & 671 & $0.55(0.42,0.69)$ & & & $\diamond$ \\
\hline \multicolumn{11}{|c|}{$\begin{array}{l}\text { Heterogeneity: } X^{2}=52.05, \text { d.f. }=3(p<0.00001), I^{2}=94 \% \\
\text { Test for overall effect: } Z=8.03(p<0.00001)\end{array}$} \\
\hline \multicolumn{11}{|c|}{ Lacunar vs. non-stroke (chronic) } \\
\hline $\begin{array}{l}\text { Hassan [12], } 2004 \\
\text { (>2 months) }\end{array}$ & 14.5 & 5.215 & 172 & 12 & 4.053 & 172 & $0.53(0.32,0.75)$ & & & t- \\
\hline $\begin{array}{l}\text { Khan [9], } 2007 \\
\text { (>3 months) }\end{array}$ & 15.14 & 5.59 & 47 & 12.49 & 4.15 & 38 & $0.53(0.09,0.96)$ & & & - \\
\hline $\begin{array}{l}\text { Pavlovic [45], } 2011 \\
\text { (1-6 months) }\end{array}$ & 14.4 & 5 & 95 & 8.9 & 3.9 & 41 & $1.16(0.77,1.56)$ & & & -- \\
\hline Subtotal $(95 \% \mathrm{CI})$ & & & 314 & & & 251 & $0.66(0.48,0.83)$ & & & $\diamond$ \\
\hline \multicolumn{11}{|c|}{$\begin{array}{l}\text { Heterogeneity: } \chi^{2}=8.02, \text { d.f. }=2(p=0.02), I^{2}=75 \% \\
\text { Test for overall effect: } Z=7.42(p<0.00001)\end{array}$} \\
\hline \multicolumn{11}{|l|}{ Lacunar vs. AT (acute) } \\
\hline Parnetti [42], 2004 (NS) & 13.9 & 5.4 & 50 & 17.8 & 13.5 & 43 & $-0.39(-0.80,0.02)$ & & & \\
\hline Beer [35], 2011 (3 days) & 10.1 & 3.6 & 25 & 9.9 & 4.9 & 19 & $0.05(-0.55,0.64)$ & & - & - \\
\hline $\begin{array}{l}\text { Khan }[9], 2008 \\
\text { ( } 2-3 \text { days) }\end{array}$ & 16.2 & 11.6 & 152 & 11.6 & 4.8 & 40 & $0.43(0.08,0.78)$ & & $-1-$ & - \\
\hline $\begin{array}{l}\text { Eikelboom }[11], 2000 \\
(<7 \text { days) }\end{array}$ & 12.7 & 5.577 & 68 & 14.1 & 6.75 & 63 & $-0.23(-0.57,0.12)$ & & & \\
\hline Subtotal (95\% CI) & & & 295 & & & 165 & $-0.02(-0.22,0.18)$ & & 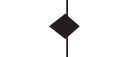 & \\
\hline \multicolumn{11}{|c|}{$\begin{array}{l}\text { Heterogeneity: } x^{2}=10.89, \text { d.f. }=3(p=0.01), I^{2}=72 \% \\
\text { Test for overall effect: } Z=0.21(p=0.83)\end{array}$} \\
\hline \multicolumn{11}{|l|}{ Lacunar vs. CE (acute) } \\
\hline Parnetti [42], 2004 (NS) & 13.9 & 5.4 & 50 & 13 & 2.5 & 31 & $0.20(-0.25,0.65)$ & & - & - \\
\hline Beer [35], 2011 (3 days) & 10.1 & 3.6 & 25 & 10.2 & 3.9 & 43 & $-0.03(-0.52,0.47)$ & & - & \\
\hline $\begin{array}{l}\text { Khan }[9], 2008 \\
\text { ( } 2-3 \text { days) }\end{array}$ & 16.2 & 11.6 & 152 & 14.3 & 6.3 & 72 & $0.19(-0.10,0.47)$ & & $f-$ & \\
\hline $\begin{array}{l}\text { Eikelboom }[11], 2000 \\
(<7 \text { days })\end{array}$ & 12.7 & 5.577 & 68 & 11.6 & 4.826 & 45 & $0.21(-0.17,0.58)$ & & $t=-$ & \\
\hline Subtotal $(95 \%$ CI) & & & 295 & & & 191 & $0.16(-0.02,0.35)$ & & $\theta$ & \\
\hline \multirow{2}{*}{\multicolumn{7}{|c|}{$\begin{array}{l}\text { Heterogeneity: } \chi^{2}=0.66, \text { d.f. }=3(p=0.88), I^{2}=0 \% \\
\text { Test for overall effect: } Z=1.71(p=0.09)\end{array}$}} & $\begin{aligned} \\
-2\end{aligned}$ & $\begin{aligned} 1 \\
-1\end{aligned}$ & 0 & 1 \\
\hline & & & & & & & & $\begin{array}{l}\text { ver } \\
\text { unar }\end{array}$ & & $\begin{array}{l}\text { Higher } \\
\text { in lacuna }\end{array}$ \\
\hline
\end{tabular}

Fig. 3. Forest plot - homocysteine: SMD of blood markers in lacunar stroke versus non-stroke controls and versus non-lacunar stroke controls at different times after stroke. AT = Atherothrombotic; $\mathrm{CE}=$ cardioembolic; NS = not stated. 


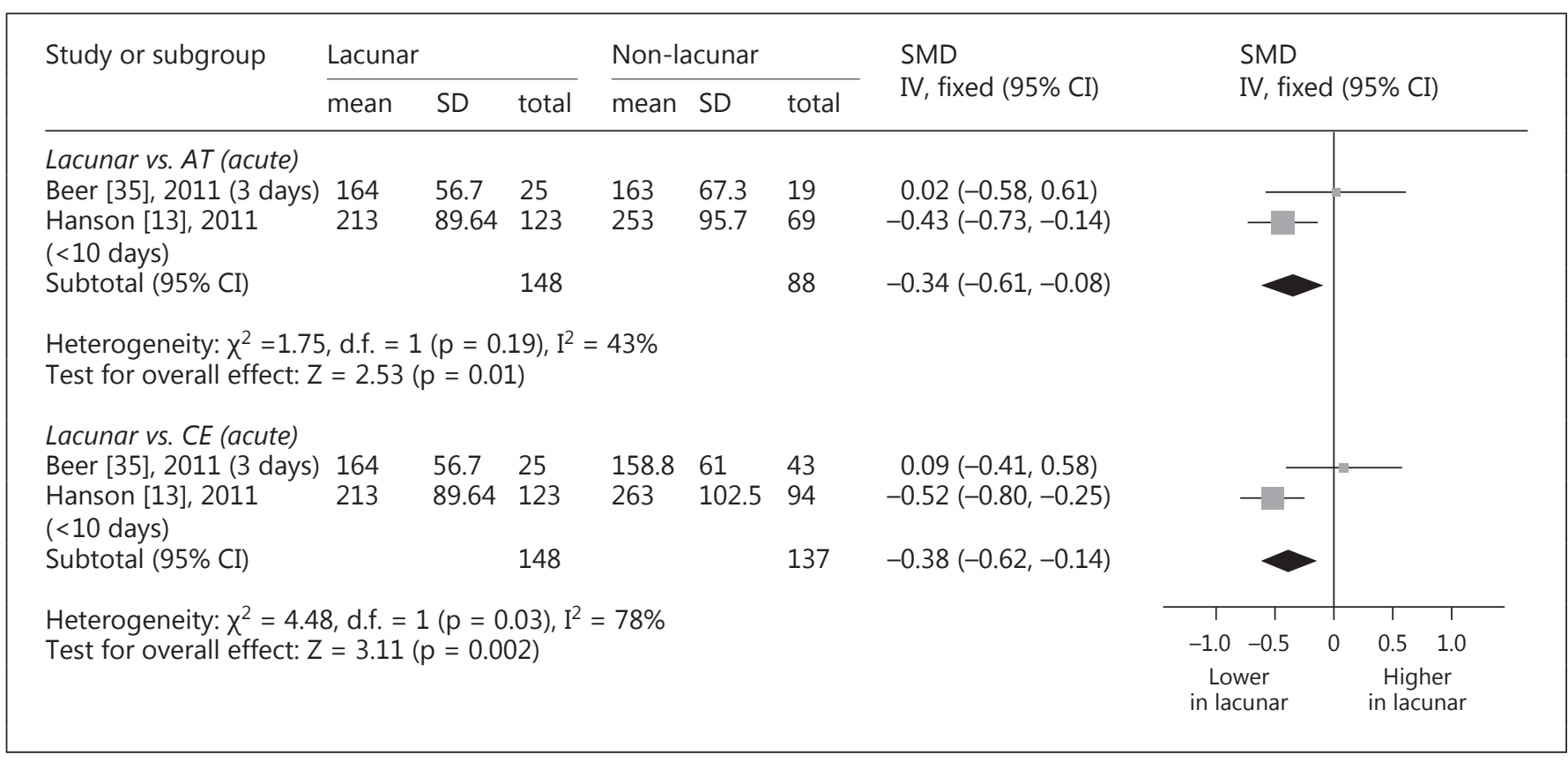

Fig. 4. Forest plot - vWF: SMD of blood markers in lacunar stroke versus non-lacunar stroke controls at different times after stroke. $\mathrm{AT}=$ Atherothrombotic; $\mathrm{CE}=$ cardioembolic.

found D-dimer significantly lower in lacunar versus cardioembolic stroke acutely (in agreement with the metaanalysis).

\section{Endothelial Activation/Dysfunction}

Homocysteine. Overall, we included 9 studies [9, 11, $12,29,35,42-45]$ (747 lacunar strokes) of which 8 could be meta-analysed (fig. 3). Homocysteine was significantly higher in lacunar stroke versus non-stroke controls, both acutely [SMD $0.55(0.42,0.69)]$ and chronically. Studies comparing lacunar to non-lacunar stroke drew blood acutely only and found no difference [atherothrombotic SMD $-0.02(-0.22,0.18)$; cardioembolic SMD $0.16(-0.02,0.35)]$. In 1 study [29] not included in the meta-analysis no difference was found in homocysteine between lacunar and non-lacunar stroke acutely.

von Willebrand Factor. We included 6 studies $[13,27$, $31,35,46,47]$ (293 lacunar strokes) of which $2[13,35]$ were meta-analysable (fig. 4). vWF was significantly higher in lacunar stroke versus non-stroke controls acutely. vWF was significantly lower in lacunar versus non-lacunar stroke [atherothrombotic SMD $-0.34(-0.61,-0.08)$; cardioembolic SMD $-0.38(-0.62,-0.14)]$ acutely. The 4 non-meta-analysable $[27,31,46,47]$ studies show conflicting results (online suppl. table 1).
E-Selectin. Here, 4 studies were included [27, 28, 35, 46] (130 lacunar strokes) but available data did not permit meta-analysis; 1 study [46] found E-selectin significantly higher in lacunar stroke versus non-stroke controls acutely but not at 1 month. Individual study reports suggest no difference between lacunar and non-lacunar stroke.

P-Selectin. A total of 7 studies were included $[27,28$, $30,31,46,48,49$ ] (227 lacunar strokes) but available data did not permit meta-analysis. P-selectin was significantly higher in lacunar versus non-stroke acutely in 2 studies $[46,49]$ but 1 study [30] found no difference. Another study [48] found P-selectin significantly lower in lacunar versus atherothrombotic stroke acutely but all other studies found no difference or did not report significance levels. Tsai et al. [48] continued to show levels to be significantly lower at 1 month but this difference disappeared at 3 months. Only 1 study [31] found P-selectin significantly lower in lacunar versus cardioembolic stroke acutely; all other studies found no difference or did not report significance levels.

Intercellular Adhesion Molecule-1. We included 5 studies [21, 27, 28, 50, 51] (344 lacunar strokes) but available data did not permit meta-analysis. In 1 study [21] significantly higher ICAM was found in lacunar 


\begin{tabular}{|c|c|c|c|c|c|c|c|c|c|}
\hline \multirow[t]{2}{*}{ Study or subgroup } & \multicolumn{3}{|c|}{ Lacunar } & \multicolumn{3}{|c|}{ Non-lacunar } & \multirow{2}{*}{$\begin{array}{l}\text { SMD } \\
\text { IV, fixed (95\% CI) }\end{array}$} & \multirow{2}{*}{\multicolumn{2}{|c|}{$\begin{array}{l}\text { SMD } \\
\text { IV, fixed ( } 95 \% \text { CI) }\end{array}$}} \\
\hline & mean & SD & total & mean & SD & total & & & \\
\hline \multicolumn{10}{|c|}{ Lacunar vs. non-stroke (acute) } \\
\hline Domac [22], 2007 (12 h) & 43.8 & 18.5 & 19 & 15.1 & 4.9 & 22 & $2.15(1.37,2.94)$ & & - \\
\hline $\begin{array}{l}\text { Guldiken [56], } 2008 \\
(<72 \text { h) }\end{array}$ & 8.79 & 3.73 & 16 & 4.68 & 3 & 23 & $1.21(0.52,1.91)$ & & $-\square$ \\
\hline $\begin{array}{l}\text { Beamer [23], } 1995 \\
\text { (4 days) }\end{array}$ & 2.8 & 2.8 & 23 & 1 & 0.9 & 20 & $0.83(0.20,1.45)$ & & $-\square-$ \\
\hline Subtotal (95\% CI) & & & 58 & & & 65 & $1.30(0.90,1.70)$ & & $\diamond$ \\
\hline \multicolumn{10}{|c|}{$\begin{array}{l}\text { Heterogeneity: } \chi^{2}=6.79, \text { d.f. }=2(p=0.03), I^{2}=71 \% \\
\text { Test for overall effect: } Z=6.35(p<0.00001)\end{array}$} \\
\hline \multicolumn{10}{|l|}{ Lacunar vs. AT (acute) } \\
\hline Nakase [53], 2008 (ON) & 3 & 3.7 & 42 & 3.3 & 3.1 & 20 & $-0.08(-0.62,0.45)$ & & \\
\hline Domac [22], 2007 (12 h) & 43.8 & 18.5 & 19 & 34.1 & 17.05 & 16 & $0.53(-0.15,1.21)$ & & - \\
\hline Vila [54], $2000(<24 \mathrm{~h})$ & 9.6 & 9.3 & 33 & 24.5 & 14.2 & 84 & $-1.14(-1.57,-0.71)$ & $-1-$ & \\
\hline $\begin{array}{l}\text { Guldiken [56], } 2008 \\
(<72 \text { h) }\end{array}$ & 8.79 & 3.73 & 16 & 4.28 & 3.97 & 12 & $1.14(0.33,1.96)$ & & - \\
\hline $\begin{array}{l}\text { Beamer [23], } 1995 \\
\text { (4 days) }\end{array}$ & 2.8 & 2.8 & 23 & 5.4 & 2.7 & 12 & $-0.92(-1.65,-0.18)$ & -- & \\
\hline Subtotal (95\% CI) & & & 133 & & & 144 & $-0.37(-0.63,-0.10)$ & $\bullet$ & \\
\hline \multicolumn{10}{|c|}{$\begin{array}{l}\text { Heterogeneity: } \chi^{2}=35.51, \text { d.f. }=4(p<0.00001), I^{2}=89 \% \\
\text { Test for overall effect: } Z=2.73(p=0.006)\end{array}$} \\
\hline \multicolumn{10}{|l|}{ Lacunar vs. CE (acute) } \\
\hline Nakase [53], 2008 (ON) & 3 & 3.7 & 42 & 3.6 & 2.6 & 30 & $-0.18(-0.65,0.29)$ & -1 & \\
\hline Vila [54], $2000(<24$ h) & 9.6 & 9.3 & 33 & 18 & 16.4 & 76 & $-0.57(-0.99,-0.15)$ & $\ldots$ & \\
\hline $\begin{array}{l}\text { Beamer }[23], 1995 \\
\text { (4 days) }\end{array}$ & 2.8 & 2.8 & 23 & 9.9 & 6.6 & 6 & $-1.82(-2.84,-0.79)$ & & \\
\hline Subtotal (95\% CI) & & & 98 & & & 112 & $-0.52(-0.82,-0.22)$ & $\bullet$ & \\
\hline \multicolumn{8}{|c|}{$\begin{array}{l}\text { Heterogeneity: } \chi^{2}=8.14, \text { d.f. }=2(p=0.02), I^{2}=75 \% \\
\text { Test for overall effect: } Z=3.40(p=0.0007)\end{array}$} & $\begin{array}{cc} & \\
-4 & -2 \\
\text { Lower } \\
\text { in lacunar }\end{array}$ & $\begin{array}{c}\mid \\
2 \\
\text { Higher } \\
\text { in lacunar }\end{array}$ \\
\hline
\end{tabular}

Fig. 5. Forest plot - IL-6: SMD of blood markers in lacunar stroke versus non-stroke controls and versus non-lacunar stroke controls at different times after stroke. $\mathrm{AT}=$ Atherothrombotic; $\mathrm{CE}=$ cardioembolic; $\mathrm{ON}=$ on admission.

stroke versus non-stroke controls acutely; another study [50] found ICAM significantly higher in lacunar versus non-stroke controls chronically. There was no difference between lacunar and other stroke subtypes acutely and no data for lacunar versus non-lacunar stroke chronically.

Vascular Cellular Adhesion Molecule-1. Here, 3 studies were included [27, 28, 51] (121 lacunar strokes) but available data did not permit meta-analysis. Results suggested no difference between lacunar stroke and non-stroke acutely and no difference between lacunar and non-lacunar stroke acutely. No studies measured VCAM chronically.

Review of Blood Markers in Lacunar Stroke

\section{Inflammation}

C-Reactive Protein. We included 8 studies $[29,34,35$, $39,47,49,52,53$ ] (490 lacunar strokes) but available data did not permit meta-analysis; 2 studies $[39,52]$ provided just over $50 \%$ of the data. CRP was higher in lacunar stroke versus non-stroke acutely in 3 studies [34, 49, 52], significantly so in 2, and the other [34] did not report if the higher value was significant. CRP was significantly lower in lacunar versus atherothrombotic stroke in 1 study [34] acutely; all other studies reported no difference (or did not state significance) between lacunar and nonlacunar stroke, acutely and chronically. 
Tumour Necrosis Factor-alpha. Overall, 5 studies were included [21, 22, 27, 47, 53] (252 lacunar strokes) but available data did not permit meta-analysis; 1 study [21] provides $45 \%$ of the data. In 2 studies [21, 22] levels of TNF- $\alpha$ were found to be significantly higher in lacunar stroke versus non-stroke controls acutely. TNF- $\alpha$ was significantly lower in $2[27,47]$ and no different in $2[22,53]$ studies reporting on lacunar versus non-lacunar stroke acutely. No studies measured TNF- $\alpha$ chronically.

Interleukin-6. A total of 9 studies were included [21$23,27,47,53-56]$ (340 lacunar strokes) of which 5 could be meta-analysed (fig. 5). IL-6 was significantly higher in lacunar stroke versus non-stroke acutely [SMD 1.3 (0.9, 1.7)], and significantly lower in lacunar versus non-lacunar stroke [atherothrombotic SMD $-0.37(-0.63,-0.10)$; cardioembolic SMD $-0.52(-0.82,-0.22)]$ acutely.

In 1 non-meta-analysable study [21] IL-6 was found to be significantly higher in lacunar stroke versus nonstroke acutely, in agreement with the meta-analysed studies; 2 non-meta-analysable studies [27, 47] found IL-6 significantly lower in lacunar versus non-lacunar stroke acutely, also in agreement with the meta-analysed studies.

The only long-term study [55] found no difference between lacunar stroke and non-stroke controls from a wholly female cohort measured 3.5 years after stroke.

\section{Discussion}

This review assessed blood markers of coagulation, fibrinolysis, endothelial dysfunction and inflammation in lacunar stroke versus non-stroke controls and other ischaemic stroke subtypes. While many markers were higher in lacunar stroke than in non-stroke controls, they were mostly lower in lacunar versus non-lacunar stroke. A brief summary follows.

\section{Coagulation/Fibrinolysis}

tPA/PAI were significantly higher in lacunar stroke versus non-stroke, acutely and chronically, and did not differ between lacunar and non-lacunar stroke, acutely or chronically.

Fibrinogen did not differ between lacunar stroke and non-stroke acutely, although we only used a single time point from Kataoka et al. [32] (bloods drawn at $<24 \mathrm{~h}$ ). If we consider their second sample (at 7 days) as a further acute measurement, lacunar stroke tends towards being significantly higher than non-stroke. Fibrinogen was significantly higher in lacunar stroke versus non-stroke chronically. Fibrinogen was significantly lower in lacu- nar versus non-lacunar stroke, acutely and chronically. However, studies excluded from the meta-analysis tended to show no overall difference between lacunar and non-lacunar stroke and chronic data came from a single study.

D-dimer was significantly higher in lacunar stroke versus non-stroke, acutely and chronically, and significantly lower in lacunar versus non-lacunar stroke, acutely and chronically.

\section{Endothelial Dysfunction}

Homocysteine was significantly higher in lacunar stroke versus non-stroke, acutely and chronically, but did not differ between lacunar and non-lacunar stroke acutely (but there were no chronic phase studies).

vWF was significantly higher in lacunar stroke versus non-stroke, acutely, with conflicting evidence chronically. vWF was significantly lower in lacunar than non-lacunar stroke acutely ( 2 studies), with conflicting but nonmeta-analysable evidence in other studies both acutely and chronically.

E-selectin was significantly higher in lacunar stroke versus non-stroke acutely (only 1 study) but not chronically and did not differ between lacunar and non-lacunar stroke, either acutely or chronically (only 1 study).

P-selectin was significantly higher in lacunar stroke versus non-stroke acutely in some but not all studies, and in the only study that reported a chronic measurement. P-selectin did not differ between lacunar and non-lacunar stroke, either acutely or chronically (only 1 study).

ICAM was significantly higher in lacunar stroke versus non-stroke, acutely and chronically (only 1 study), and did not differ between lacunar and non-lacunar stroke acutely (with no studies chronically).

VCAM did not differ between lacunar stroke and nonstroke nor between lacunar and non-lacunar stroke acutely. There were no studies chronically.

\section{Inflammation}

CRP was significantly higher in lacunar stroke versus non-stroke, acutely and chronically (only 1 study) and did not differ between lacunar and non-lacunar stroke acutely or chronically (only 1 study).

TNF- $\alpha$ was significantly higher in lacunar stroke versus non-stroke acutely with no studies chronically. There was conflicting evidence on levels of TNF- $\alpha$ in lacunar versus non-lacunar stroke acutely with no studies chronically.

IL-6 was significantly higher in lacunar stroke versus non-stroke acutely but did not differ chronically. IL- 6 was
Wiseman/Marlborough/Doubal/Webb/ Wardlaw 
significantly lower in lacunar versus non-lacunar stroke acutely, but there were no chronic phase studies.

This suggests that plasma marker elevation in lacunar stroke is likely to reflect the process of having a stroke rather than that systemic inflammation or endothelial dysfunction is specific to lacunar stroke. The available data were limited and do not exclude the possibility that peripheral inflammatory or endothelial dysfunction processes are associated with lacunar stroke specifically.

There were limitations to the studies. Most were small, with varying methods and an inconsistent definition of 'lacunar stroke', as highlighted previously [57]. Papers reviewed used the term lacunar stroke to reflect a clinical entity, i.e. clinical presentation with a stroke, but definitions varied and we refer readers to the new neuroimaging standards [10]. We were not able to differentiate different mechanisms of lacunar stroke. Most lacunar strokes are due to recent small subcortical infarcts, and most of these relate to intrinsic small vessel disease. However, they also arise from atherothromboembolism (large artery) or cardioembolism in a small proportion of patients and it was not possible to differentiate these cases.

There was heterogeneity across several aspects of the methods. Many used TOAST [19] but as this uses risk factors to categorize patients it potentially introduces classification bias. A patient with an unclear diagnosis of lacunar stroke but concurrent hypertension or diabetes might (rightly or wrongly) be classified as 'lacunar' using this system, although hypertension and diabetes were equally prevalent risk factors between ischaemic stroke subtypes in 21,980 stroke patients when subtypes were classified without risk factors [58]. Several did not report on whether their findings achieved statistical significance; in the absence of an explicit statement, we report this as 'not stated'. In some studies blood was drawn after overnight fasting, whereas in others non-fasting blood was collected. Studies used different units of measurement and assay methods. None reported on whether the patients had recent infection or neutrophilia, or if these patients were excluded. Timing of blood draw in relation to stroke varied but is important to account for each marker's individual 'response curve' which changes over time. Fassbender et al. [59] found levels of IL-6 to rise rapidly following onset of ischaemic stroke, reaching a plateau at 10 h until 3 days before returning to normal by day 7 . They did not subtype stroke and hence their study was not included in this review. Between-study heterogeneity on time to blood draw complicates subsequent analysis, although meta-analyses use within-study data and so will have minimized any effect of between-study variation.
Our review had limitations. We did not study markers in cerebrospinal fluid. We did not review the association of marker levels with lesion size or clinical outcome as data were sparse. Ahmad et al. [60] found markers of neuronal damage correlated with infarct size, which might explain why marker levels in non-lacunar stroke were frequently higher than in lacunar stroke in the acute phase. We were not able to analyse differences between groups reported as top versus bottom quantiles.

Our review had strengths, including assessment of differences between stroke subtypes, quality assessment of included studies, meticulous extraction of data and metaanalysis thereof, wherever suitable data were available. Previous reviews compared lacunar stroke to non-stroke controls only and therefore did not distinguish lacunar stroke specifically from stroke in general.

To determine if there is a difference in coagulation, fibrinolysis, endothelial dysfunction and inflammation in lacunar versus other stroke subtypes requires a large prospective study of blood markers in accurately phenotyped patients with lacunar versus non-lacunar stroke classified using non-risk-factor-based definitions. Future studies should clearly define and diagnose lacunar stroke, avoid subtyping stroke using risk factor-based classifications, explicitly report negative findings and significance levels, and obtain blood several weeks post-stroke to avoid confounding from an acute phase response.

\section{Appendix}

Search Strategy.

(1) brain ischemia/ or brain infarction/ or brain stem infarctions/ or cerebral infarction/ or hypoxia-ischemia, brain/ or stroke/

(2) (isch?emi\$ adj6 (stroke\$ or apoplex\$ or cerebral vasc\$ or cerebrovasc $\$$ or cva or attack\$)).tw.

(3) ((brain or cerebr\$ or cerebell\$ or vertebrobasil\$ or hemispher\$ or intracran\$ or intracerebral or infratentorial or supratentorial or middle cerebr\$ or mca $\$$ or anterior circulation) adj5 (isch?emi\$ or infarct\$ or thrombo $\$$ or emboli\$ or occlus\$ or hypoxi\$)).tw.

(4) 1 or 2 or 3

(5) (lacun\$ or small vessel\$ or small infarct\$ or microinfarct\$ or subcortical lesion\$ or subcortical infarct\$ or microvascular\$ or microcirculation\$).tw.

(6) 4 and 5

(7) blood-brain barrier/ or endothel\$, vascular/ or tunica inti$\mathrm{ma} /$ or microcirculation/

(8) (endotheli\$ adj5 (function\$ or dysfunction\$ or impairment\$)).tw.

(9) (endogenous tissue plasminogen activator or endogenous tPA).tw 
(10) thrombosis.tw

(11) fibrinogen.tw

(12) fibrinolysis.tw

(13) homocysteine.tw

(14) (ICAM or Intra cellular adhesion molecule).tw

(15) (VCAM or Vascular Cell Adhesion Molecule).tw

(16) (IL6 or Interleukin 6).tw

(17) (CRP or C reactive protein).tw

(18) von Willebrand factor.tw

(19) plasminogen activator inhibitor.tw

(20) selectin $\$ . t w$

(21) D-dimer.tw

(22) (TNF or TNF-a or TNF-alpha or TNF-a or tumo?r necrosis factor alpha).tw

(23) or/7-22

(24) 6 and 23

(25) limit 24 to humans

\section{Acknowledgements}

The authors acknowledge Dr. Stephen Makin for assistance in collecting some of the data. Stewart Wiseman is supported by a Principal's Career Development PhD Scholarship from the University of Edinburgh.

\section{Disclosure Statement}

None.

\section{References}

1 Knottnerus IL, Ten Cate H, Lodder J, Kessels F, Van Oostenbrugge RJ: Endothelial dysfunction in lacunar stroke: a systematic review. Cerebrovasc Dis 2009;27:519-526.

-2 Stevenson SF, Doubal FN, Shuler K, Wardlaw JM: A systematic review of dynamic cerebral and peripheral endothelial function in lacunar stroke versus controls. Stroke 2010; 41:e434-e442.

3 Hasan N, McColgan P, Bentley P, Edwards RJ Sharma P: Towards the identification of blood biomarkers for acute stroke in humans: a comprehensive systematic review. $\mathrm{Br} \mathrm{J}$ Clin Pharmacol 2012;74:230-240.

-4 Moher D, Liberati A, Tetzlaff J, Altman DG: Preferred reporting items for systematic reviews and meta-analyses: the PRISMA statement. PLoS Med 2009;6:e1000097.

5 Stroup DF, Berlin JA, Morton SC, Olkin I, Williamson GD, Rennie D, Moher D, Becker BJ, Sipe TA, Thacker SB: Meta-analysis of observational studies. JAMA 2000;283:20082012.

6 Mischak H, Allmaier G, Apweiler R, Attwood $\mathrm{T}$, Baumann M, Benigni A, Bennett SE, Bischoff R, Bongcam-Rudloff E, Capasso G, Coon JJ, D'Haese P, Dominiczak AF, Dakna M, Dihazi H, et al: Recommendations for biomarker identification and qualification in clinical proteomics. Sci Transl Med 2010;2: $46 \mathrm{ps} 42$.

7 McShane LM, Altman DG, Sauerbrei W, Taube SE, Gion M, Clark GM: Reporting Recommendations for Tumour Marker Prognostic Studies (REMARK). Eur J Cancer 2005;41: 1690-1696.

-8 Von Elm E, Altman DG, Egger M, Pocock SJ, Gøtzsche PC, Vandenbroucke JP: The Strengthening the Reporting of Observational Studies in Epidemiology (STROBE) statement: guidelines for reporting observational studies. Rev Esp Salud Publica 2007;82:251259.

-9 Khan U, Crossley C, Kalra L, Rudd A, Wolfe CDA, Collinson P, Markus HS: Homocysteine and its relationship to stroke subtypes in a UK black population: the south London ethnicity and stroke study. Stroke 2008;39:29432949.

10 Wardlaw JM, Smith EE, Biessels GJ, Cordonnier C, Fazekas F, Frayne R, Lindley RI, O'Brien JT, Barkhof F, Benavente OR, Black SE, Brayne C, Breteler M, Chabriat H, Decarli $C$, et al: Neuroimaging standards for research into small vessel disease and its contribution to ageing and neurodegeneration. Lancet Neurol 2013;12:822-838.

11 Eikelboom JW, Hankey GJ, Anand SS, Lofthouse E, Staples N, Baker RI: Association between high homocyst(e)ine and ischemic stroke due to large- and small-artery disease but not other etiologic subtypes of ischemic stroke. Stroke 2000;31:1069-1075.

12 Hassan A, Hunt BJ, O'Sullivan M, Bell R, D’Souza R, Jeffery S, Bamford JM, Markus HS: Homocysteine is a risk factor for cerebral small vessel disease, acting via endothelial dysfunction. Brain 2004;127:212-219.

13 Hanson E, Jood K, Karlsson S, Nilsson S, Blomstrand C, Jern C: Plasma levels of von Willebrand factor in the etiologic subtypes of ischemic stroke. J Thromb Haemost 2011;9: 275-281.

14 Higgins J, Greens S: Cochrane Handbook for Systematic Reviews of Interventions, version 5.1.0 (updated March 2011). The Cochrane Collaboration, 2011 (available from www.cochrane-handbook.org).

15 Iso H, Moriyama Y, Sato S, Kitamura A, Tanigawa $\mathrm{T}$, Yamagishi $\mathrm{K}$, Imano $\mathrm{H}$, Ohira $\mathrm{T}$, Okamura T, Naito Y, Shimamoto T: Serum total homocysteine concentrations and risk of stroke and its subtypes in Japanese. Circulation 2004;109:2766-2772.

16 Ohira T, Shahar E, Chambless LE, Rosamond WD, Mosley TH, Folsom AR: Risk factors for ischemic stroke subtypes: the Atherosclerosis Risk in Communities study. Stroke 2006;37: 2493-2498.

17 Andersson J, Johansson L, Ladenvall P, Wiklund P-G, Stegmayr B, Jern C, Boman K: $\mathrm{C}$-reactive protein is a determinant of firstever stroke: prospective nested case-referent study. Cerebrovasc Dis 2009;27:544-551.

18 Chei C-L, Yamagishi K, Kitamura A, Kiyama M, Imano H, Ohira T, Tanigawa T, Sankai T, Ishikawa $Y$, Sato $\mathrm{S}$, Iso $\mathrm{H}$ : $\mathrm{C}$-reactive protein levels and risk of stroke and its subtype in Japanese: the Circulatory Risk in Communities Study (CIRCS). Atherosclerosis 2011;217:187-193.

19 Adams HP, Bendixen BH, Kappelle LJ, Biller J, Love BB, Gordon DL, Marsh EE: Classification of subtype of acute ischemic stroke. Definitions for use in a multicenter clinical trial. TOAST. Trial of Org 10172 in Acute Stroke Treatment. Stroke 1993;24:35-41.

20 Kilpatrick TJ, Matkovic Z, Davis SM, McGrath CM, Dauer RJ: Hematologic abnormalities occur in both cortical and lacunar infarction. Stroke 1993;24:1945-1950.

21 Castellanos M, Castillo J, Garcia MM, Leira R, Serena J, Chamorro A, Davalos A: Inflammation-mediated damage in progressing lacunar infarctions: a potential therapeutic target. Stroke 2002;33:982-987.

22 Domac FM, Somay G, Misirli H, Erenoglu NY: Tumor necrosis factor alpha serum levels and inflammatory response in acute ischemic stroke. Neurosciences 2007;12:25-30.

23 Beamer NB, Coull BM, Clark WM, Hazel JS, Silberger JR: Interleukin-6 and interleukin-1 antagonist in acute stroke. Ann Neurol 1995; 37:800-805. 
-24 Lindgren A, Lindoff C, Norrving B, Astedt B, Johansson BB: Tissue plasminogen activator and plasminogen activator inhibitor-1 in stroke patients. Stroke 1996;27:1066-1071.

-25 Salobir B, Sabovic M, Peternel P, Stegnar M, Grad A: Classic risk factors, hypercoagulability and migraine in young women with cerebral lacunar infarctions. Acta Neurol Scand 2002;105:189-195.

26 Jood K, Ladenvall P, Tjärnlund-Wolf A, Ladenvall C, Andersson M, Nilsson S, Blomstrand C, Jern C: Fibrinolytic gene polymorphism and ischemic stroke. Stroke 2005;36: 2077-2081.

-27 Tuttolomondo A, Di Sciacca R, Di Raimondo D, Serio A, D’Aguanno G, La Placa S, Pecoraro R, Arnao V, Marino L, Monaco S, Natalè E, Licata G, Pinto A: Plasma levels of inflammatory and thrombotic/fibrinolytic markers in acute ischemic strokes: relationship with TOAST subtype, outcome and infarct site. J Neuroimmunol 2009;215:84-89.

-28 Tuttolomondo A, Pinto A, Corrao S, Di Raimondo D, Fernandez P, Di Sciacca R, Arnao $\mathrm{V}$, Licata G: Immuno-inflammatory and thrombotic/fibrinolytic variables associated with acute ischemic stroke diagnosis. Atherosclerosis 2009;203:503-508.

29 Yokokawa H, Goto A, Terui K, Funami Y, Watanabe K, Yasumura S: Prevalence of metabolic syndrome and serum marker levels in patients with four subtypes of cerebral infarction in Japan. J Clin Neurosci 2008;15:769773.

-30 Ilhan D, Ozbabalik D, Gulcan E, Ozdemir O, Gülbaçs Z: Evaluation of platelet activation, coagulation, and fibrinolytic activation in patients with symptomatic lacunar stroke. Neurologist 2010;16:188-191.

31 Bath PMW, Blann A, Smith N, Butterworth RJ: Von Willebrand factor, P-selectin and fibrinogen levels in patients with acute ischaemic and haemorrhagic stroke, and their relationship with stroke sub-type and functional outcome. Platelets 1998;9:155-159.

- 32 Kataoka S, Hirose G, Hori A, Shirakawa T, Saigan T: Activation of thrombosis and fibrinolysis following brain infarction. J Neurol Sci 2000;181:82-88.

- 33 Jood K, Danielson J, Ladenvall C, Blomstrand C, Jern C: Fibrinogen gene variation and ischemic stroke. J Thromb Haemost 2008;6:897904.

-34 Alvarez-Perez FJ, Castelo-Branco M, AlvarezSabin J: Usefulness of measurement of fibrinogen, D-dimer, D-dimer/fibrinogen ratio, Creactive protein and erythrocyte sedimentation rate to assess the pathophysiology and mechanism of ischaemic stroke. J Neurol Neurosurg Psychiatry 2011;82:986-992.

35 Beer C, Blacker D, Hankey GJ, Puddey IB: Association of clinical and aetiologic subtype of acute ischaemic stroke and inflammation, oxidative stress and vascular function: a crosssectional observational study. Med Sci Monit 2011;17:467-473.
36 Zhang B, Zhang W, Li X, Pu S, Yin J, Yang N, Yi Y, Gao Q, Gao C: Admission markers predict lacunar and non-lacunar stroke in young patients. Thromb Res 2011;128:14-17.

37 Takano K, Yamaguchi T, Uchida K: Markers of a hypercoagulable state following acute ischemic stroke. Stroke 1992;23:194-198.

38 Ageno W, Finazzi S, Steidl L, Biotti MG, Mera V, Melzi D’Eril G, Venco A: Plasma measurement of $\mathrm{D}$-dimer levels for the early diagnosis of ischemic stroke subtypes. Arch Intern Med 2002;162:2589-2593.

39 Montaner J, Perea-Gainza M, Delgado P, Ribó M, Chacón P, Rosell A, Quintana M, Palacios ME, Molina CA, Alvarez-Sabín J: Etiologic diagnosis of ischemic stroke subtypes with plasma biomarkers. Stroke 2008;39: 2280-2287.

40 Brouns R, Van den Bossche J, De Surgeloose D, Sheorajpanday R, De Deyn PP: Clinical and biochemical diagnosis of small-vessel disease in acute ischemic stroke. J Neurol Sci 2009;285:185-190.

41 Isenegger J, Meier N, Lämmle B, Alberio L, Fischer U, Nedeltchev K, Gralla J, Kohler H-P, Mattle HP, Arnold M: D-dimers predict stroke subtype when assessed early. Cerebrovasc Dis 2010;29:82-86.

42 Parnetti L, Caso V, Santucci a A, Corea F, Lanari A, Floridi A, Conte C, Bottiglieri T: Mild hyperhomocysteinemia is a risk factor in all etiological subtypes of stroke. Neurol Sci 2004;25:13-17.

43 Khan U, Hassan A, Vallance P, Markus HS: Asymmetric dimethylarginine in cerebral small vessel disease. Stroke 2007;38:411-413.

44 Jeong S-K, Kim D-H, Cho YI: Homocysteine and pulsatility index in lacunar infarction. Clin Neurol Neurosurg 2011;113:459-463.

45 Pavlovic AM, Pekmezovic T, Obrenovic R, Novakovic I, Tomic G, Mijajlovic M, Sternic $\mathrm{N}$ : Increased total homocysteine level is associated with clinical status and severity of white matter changes in symptomatic patients with subcortical small vessel disease. Clin Neurol Neurosurg 2011;113:711-715.

46 Kozuka K, Kohriyama T, Nomura E, Ikeda J, Kajikawa H, Nakamura S: Endothelial markers and adhesion molecules in acute ischemic stroke - sequential change and differences in stroke subtype. Atherosclerosis 2002;161: 161-168.

47 Licata G, Tuttolomondo A, Di Raimondo D, Corrao S, Di Sciacca R, Pinto A: Immuno-inflammatory activation in acute cardio-embolic strokes in comparison with other subtypes of ischaemic stroke. Thromb Haemost 2009: 929-937.
48 Tsai N-W, Chang W-N, Shaw C-F, Jan C-R, Chang H-W, Huang C-R, Chen S-D, Chuang Y-C, Lee L-H, Wang H-C, Lee T-H, Lu C-H: Levels and value of platelet activation markers in different subtypes of acute non-cardioembolic ischemic stroke. Thromb Res 2009;124: 213-218.

49 Turgut B, Turgut N, Celik Y, Tekgündüz E, Pamuk GE, Demir M: Differences in plateletleukocyte aggregates among subtypes of acute cerebral ischemia. J Neurol Sci 2011;305:126130.

50 Hassan A, Hunt BJ, O’Sullivan M, Parmar K, Bamford JM, Briley D, Brown MM, Thomas DJ, Markus HS: Markers of endothelial dysfunction in lacunar infarction and ischaemic leukoaraiosis. Brain 2003;126:424-432.

51 Supanc V, Biloglav Z, Kes VB, Demarin V: Role of cell adhesion molecules in acute ischemic stroke. Ann Saudi Med 2011;31:365370.

52 Ladenvall C, Jood K, Blomstrand C, Nilsson S, Jern C, Ladenvall P: Serum C-reactive protein concentration and genotype in relation to ischemic stroke subtype. Stroke 2006;37: 2018-2023.

53 Nakase T, Yamazaki T, Ogura N, Suzuki A, Nagata K: The impact of inflammation on the pathogenesis and prognosis of ischemic stroke. J Neurol Sci 2008;271:104-109.

54 Vila N, Castillo J, Davalos A, Chamorro A: Proinflammatory cytokines and early neurological worsening in ischemic stroke. Stroke 2000;31:2325-2329.

55 Salobir B, Sabovic M: Possible vascular-bedspecific role of interleukin-6 in young women with a history of myocardial infarction, lacunar cerebral infarction and deep vein thrombosis. Cytokine 2004;25:265-272.

56 Guldiken B, Guldiken S, Turgut B, Turgut N Demir M, Celik Y, Arikan E, Tugrul A: The roles of oxidized low-density lipoprotein and interleukin-6 levels in acute atherothrombotic and lacunar ischemic stroke. Angiology 2008;59:224-229.

57 Potter GM, Marlborough FJ, Wardlaw JM: Wide variation in definition, detection, and description of lacunar lesions on imaging. Stroke 2011;42:359-366.

58 Jackson C, Sudlow C: Are lacunar strokes really different? A systematic review of differences in risk factor profiles between lacunar and nonlacunar infarcts. Stroke 2005;36:891901.

59 Fassbender K, Rossol S, Kammer T, Daffertshofer M, Wirth S, Dollman M, Hennerici M: Proinflammatory cytokines in serum of patients with acute cerebral ischemia: kinetics of secretion and relation to the extent of brain damage and outcome of disease. J Neurol Sci 1994;122:135-139.

60 Ahmad O, Wardlaw J, Whiteley WN: Correlation of levels of neuronal and glial markers with radiological measures of infarct volume in ischaemic stroke: a systematic review. Cerebrovasc Dis 2012;33:47-54.
Review of Blood Markers in Lacunar Stroke
Cerebrovasc Dis 2014;37:64-75

DOI: $10.1159 / 000356789$ 